\title{
Red Liriope platyphylla contains a large amount of polyphenolic compounds which stimulate insulin secretion and suppress fatty liver formation through the regulation of fatty acid oxidation in OLETF rats
}

\author{
HYE RYUN LEE ${ }^{1}$, JI EUN KIM ${ }^{1}$, JUN SEO GOO $^{1}$, SUN IL CHOI $^{1}$, IN SIK HWANG ${ }^{1}$, YOUNG JU LEE ${ }^{1}$, \\ HONG JOO SON ${ }^{1}$, HEE SEOB LEE ${ }^{2}$, JONG SUP LEE ${ }^{3}$ and DAE YOUN HWANG ${ }^{1}$ \\ ${ }^{1}$ College of Natural Resources and Life Science, Pusan National University, Gyeongsangnam-do 627-706; \\ ${ }^{2}$ College of Human Ecology, Pusan National University, Busan 609-735; ${ }^{3}$ Nonghyup Sandong \\ Processing Plant, Miryang 627-706, Republic of Korea
}

Received May 9, 2012; Accepted July 3, 2012

DOI: $10.3892 /$ ijmm.2012.1081

\begin{abstract}
Red Liriope platyphylla (RLP) manufactured by two repeated steps (steaming and drying) stimulates the insulin secretion ability and glucose receptor signaling pathway in an animal model for type I diabetes. This study examined the levels of glucose and lipid metabolism-related factors in a useful animal model for type II diabetes with obesity following RLP treatment for 3 weeks to determine if RLP treatment affects the glucose concentration, insulin secretion and fatty acid oxidation. The following results were obtained: i) RLP contained a large amount of polyphenolic compounds; ii) insulin secretion was induced in RLP-treated OLETF rats, although there were no significant differences in body weight, glucose tolerance test and glucose concentration; iii) the RLP-treated OLETF rats showed a significant increase in adiponectin concentration but the concentration of triglyceride and LDL decreased compared to the vehicle-treated rats; iv) although the abdominal fat mass and adipocyte size did not change with RLP treatment, expression of the adipocyte marker genes and $\beta$-oxidation genes in fat tissue was recovered to the level of the LETO rats; v) fatty liver formation was reduced dramatically in the liver of the RLP-treated group compared to the vehicle-treated group; vi) the expression of adipocyte marker genes and the $\beta$-oxidation gene in the liver tissue were generally similar to those of the abdominal fat but PPAR- $\gamma$ showed a reverse pattern in the RLP- and vehicletreated OLETF rats. These results suggest that RLP may
\end{abstract}

Correspondence to: Professor Dae Youn Hwang, Department of Biomaterials Science, College of Natural Resources and Life Science, Pusan National University, 50 Cheonghak-ri, Samnangjin-eup Miryang-si, Gyeongsangnam-do 627-706, Republic of Korea

E-mail: dyhwang@pusan.ac.kr

Key words: red Liriope platyphylla, OLETF rat, insulin, adiponectin, fatty liver stimulate insulin secretion and a decrease in lipid in serum, and may also suppress fatty liver formation through the regulation of fatty acid oxidation. The data presented here highlight the possibility that RLP can be considered a candidate for the prevention or alleviation of obesity-related diseases.

\section{Introduction}

Liriope platyphylla (LP) is a perennial seed-reproducing plant that is distributed widely throughout the temperate climate region of the northern hemisphere. In Korea, these plants grow principally in low mountain areas at altitudes $<500 \mathrm{~m}$ above sea level, and their leaves remain green throughout the year (1). LP is a herb that has been used for a long time in oriental medicine to treat asthma as well as bronchial and lung inflammation (2). The effects of LP root extracts on the prevention of obesity, diabetes, inflammation and neurodegenerative disease have previously been demonstrated (3-7). Among these therapeutic effects, LP exhibited therapeutic potential in human subjects suffering from obesity and diabetes. In particular, Gyeongshingangjeehwan (GGEx; mainly containing LP) has been reported to prevent obesity and hypertriglyceridemia by inhibiting feeding and activating hepatic peroxisome proliferator-activated receptor- $\alpha$ in OLETF male rats (6). In addition, the homoisoflavone-enriched fraction of LP methanol extracts increased insulin-stimulated glucose uptake in 3T3-L1 adipocytes through increased glucose transporter (Glut)-4 contents in the plasma membrane (3). Some novel compounds have been isolated from LP using methanol extraction. Of these compounds, LP9M80-H induces insulin secretion and differentially regulates the expression of Glut-1 and Glut-3 expression through the mitogen-activated protein kinase (MAPK) and phosphoinisitide-3-kinase (PI3-K) signaling pathways (8). Furthermore, an aqueous extract of LP induces the upregulation of insulin secretion and the downregulation of glucose concentration through an increase in the expression of several Glut proteins regulated by the insulin signaling pathway (9). 
Steaming is often applied to medicinal plants to increase the levels or efficacy of their functional components as well as to induce chemical transformations of the specific components (10). This process has been applied most successfully to ginseng, derivations of which are taken orally as adaptogens, aphrodisiacs, nourishing stimulants, as well as in the treatment of type II diabetes and sexual dysfunction in men (11-13). Korean ginseng is found in two forms; Korean white ginseng (KWG) (Panax ginseng C.A. Meyer), which is air-dried ginseng, and Korean red ginseng (KRG) (Ginseng Radix Rubra), which is repeatedly steamed (10). During the steaming process, several important components in ginseng, such as ginsenosides, acidic polysaccharides and phenolics, are transformed into different components and several new compounds, such as non-saponinpolyaceylene, maltol and amino acid $(14,15)$. Recently, RLP was produced by a steaming process and its effects on the insulin secretion ability and insulin receptor signaling pathway were examined. The maximum insulin secretion was observed in INS cells treated with 3-SLP and 9-SALP (16). Despite these initial results, there are no reports on how RLP affects the insulin secretion ability and adipocyte hypertrophy to improve diabetes and obesity.

Therefore, in this study, we investigated the effects of RLP on insulin secretion and adipocyte hypertrophy using a type II diabetes model. Significant changes on the insulin release, lipid concentration, fatty liver formation and fatty acid oxidation-related genes were observed in the pancreas, abdominal fat and livers of OLETF rats in response to RLP treatment.

\section{Materials and methods}

Preparation of RLP. LP roots were collected from plantations in the Miryang area of Korea (Fig. 1) and dried with a hot-air drier (JSR Instruments, Uttaranchal, India) at $60^{\circ} \mathrm{C}$. The voucher specimens (WPC-11-010) were deposited at the Functional Materials Bank of PNU-Wellbeing RIS Center at Pusan National University. Six hundred grams of dry roots were reduced to powder using an electric blender. The water extract was purified at $100^{\circ} \mathrm{C}$ for $2 \mathrm{~h}$ using circulated extraction equipment (IKA Labortechnik, Staufen, Germany) after adding 2 liters of distilled water. The composition of RLP was measured as previously described $(17,18)$. Extract solutions were concentrated into dry pellets using a rotary evaporator (Eyela, Tokyo, Japan) and stored at $-80^{\circ} \mathrm{C}$ until use.

HPLC analysis of RLP. The AEtLP was analyzed by ILC 3000 HPLC system (Interface Engineering, Co., Ltd., Seoul, Korea) equipped with Corona ${ }^{\circledR} \mathrm{CAD}^{\circledR}$ Detector (ESA Biosciences, Inc., Chelmsford, MA, USA). The chromatographic separation was performed on a CapCell PAK MG C18 (4.6x250 mm, particle size $5 \mu \mathrm{m}$; Shiseido Co., Ltd., Tokyo, Japan). The mobile phase consisted of solvent A (deionized water) and solvent $\mathrm{B}$ (acetonitrile) using the gradient elution program; 0-25 min, 30-90\% of solvent B and 25-40 min, $90 \%$ of solvent B. A flow rate of $1.0 \mathrm{ml} / \mathrm{min}$ was used for the sample analysis. The nebulizer gas was compressed nitrogen. The gas flow rate and gas pressure were maintained at $1.53 \mathrm{l} / \mathrm{min}$ and $35 \pm 2 \mathrm{psi}$, respectively. The output signal of the detector was recorded using Clarity ${ }^{\mathrm{TM}}$ Chromatography Software (DataApex, Prague, Czech Republic).

Care and use of OLETF rats. All animal experimental procedures in this study were approved by the Institutional Animal Care and Use Committee (IACUC) at Pusan National University (PNU-2011-000220). The animals were handled in a Pusan National University-Laboratory Animal Resources Center accredited by the Korea FDA in accordance with the USA NIH guidelines (accredited unit no. 000996). All rats were housed in specified pathogen-free (SPF) conditions under a strict light cycle (light on at 06:00 $\mathrm{h}$ and off at 18:00 h), and given a standard irradiated chow diet (Purina Mills, Inc.) ad libitum. The 20-week-old LETO and OLETF rats used in this study were supplied by Central Laboratory Animals, Inc. (Seoul, Korea).

Treatment of RLP and detection of glucose levels. RLP was dissolved in distilled water to give a final concentration of $9.1 \mu \mathrm{g} / \mathrm{ml}$. OLETF rats were divided randomly into 2 groups. The first group received a comparable volume of daily water via oral administration (vehicle-treated group), whereas the second group received $50 \mathrm{mg} / \mathrm{kg}$ body weight/day of RLP via oral administration for 3 weeks (RLP-treated group). After 3 weeks, the glucose concentration was detected following $24 \mathrm{~h}$ of fasting using the sensitive strip of the Blood Glucose Monitoring System (i-Sens, Inc., Seoul, Korea).

Intraperitoneal glucose tolerance test. The glucose tolerance was determined from 5 rats/group using an intraperitoneal glucose tolerance test (IPGTT; $1.5 \mathrm{mg}$ glucose/g after a $24 \mathrm{~h}$ fast with blood glucose measurements being made at 0,15 , 30,60 and $120 \mathrm{~min}$ ). The serum glucose level from rats was detected using the sensitive strip of the Blood Glucose Monitoring System (i-Sens, Inc.,).

Serum biochemical analysis. After the final administration of RLP, the rats were fasted for $24 \mathrm{~h}$ and blood was collected from the abdominal vein. Serum was obtained by the centrifugation of blood incubated for $30 \mathrm{~min}$ at room temperature. The biochemical components in the serum were assayed using an Automated Serum Analyzer (Hitachi 747, Japan). All assays were measured with fresh serum and carried out in duplicate.

Quantification of insulin and adiponectin by ELISA. The concentration of insulin and adiponectin in serum from the LETO and OLETF rats was detected using an ultrasensitive assay procedure and reagents in the Mercodia Rat Insulin ELISA kit (Cat. 10-1250-01; Mercodia, Sweden) and Adiponectin ELISA kit (Cat. AG-45A-0005TP-KI01; AdipoGen, Inc., Korea). The sera and standards were incubated in a plate shaker at $100-150 \mathrm{rpm}$ at room temperature for $2 \mathrm{~h}$ on antibody-coated plates. The wells were then washed 6 times using an automatic plate washer (PV100; Hoefer, Inc., USA). HRP conjugate was added to all the plates, which were then incubated in a shaker for $15 \mathrm{~min}$ at room temperature. The reaction was quenched by adding $50 \mu \mathrm{l}$ of a stop solution $\left(0.5 \mathrm{M} \mathrm{H}_{2} \mathrm{SO}_{4}\right)$. The plates were read at $450 \mathrm{~nm}$ using a Molecular Devices Vmax plate reader (Sunnyvale, CA, USA). 
$\underline{A}$

\begin{tabular}{lcc}
\hline \multirow{2}{*}{ Component } & \multicolumn{2}{c}{ Contents (\%) } \\
\cline { 2 - 3 } Moisture & $14.20 \pm 0.08$ & RLP \\
Crude protein & $4.51 \pm 0.04$ & $8.24 \pm 0.09^{*}$ \\
Carbohydrate & $78.48 \pm 0.09$ & $4.94 \pm 0.24^{*}$ \\
Crude fat & $0.51 \pm 0.10$ & $83.22 \pm 0.37^{*}$ \\
Crude ash & $2.30 \pm 0.08$ & $0.94 \pm 0.04^{*}$ \\
\hline Total & 100 & $2.66 \pm 0.08^{*}$ \\
\hline
\end{tabular}

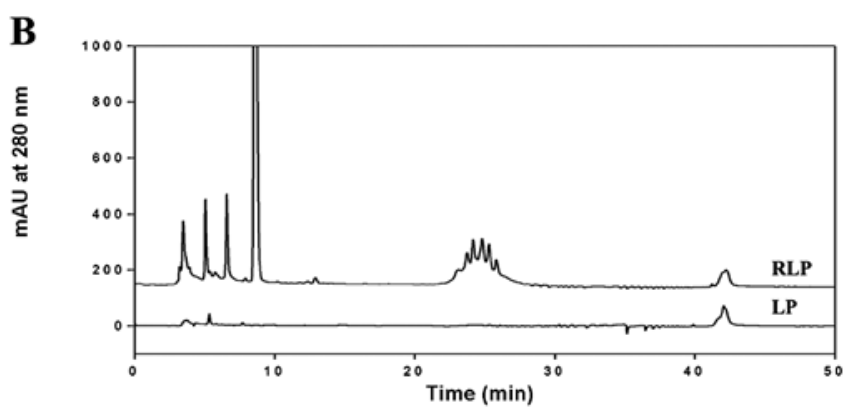

Figure 1. (A) Compositions and (B) chromatogram of RLP. The peak in the chromatogram indicates the increase of the polyphenolic compounds. Data is reported as the mean \pm SD from 3 replicates. ${ }^{*} \mathrm{P}<0.05$ is the significance level compared to the LP.

Histological analysis and immunostaining. The abdominal fat and liver collected from the rats were fixed with $10 \%$ formalin for $48 \mathrm{~h}$, embedded in paraffin wax and sectioned into $3 \mu \mathrm{m}$ slices. The fat and liver sections were then stained with hematoxylin \& eosin (H\&E; Sigma-Aldrich, St. Louis, MO, USA), and observed by optical microscopy. The size, number and morphology of the adipocytes were measured using Leica Application Suite (Leica Microsystems, Switzerland).

Immunohistochemical analysis was performed as previously described (8). Briefly, the distribution of insulin protein was observed using optical microscopy after fixing the tissue samples in $5 \%$ formalin for $48 \mathrm{~h}$, embedding the tissues in paraffin and acquiring $3 \mu \mathrm{m}$ sections. Each section was de-paraffinized with xylene, rehydrated and pretreated for $30 \mathrm{~min}$ at room temperature with a phosphate-buffered saline (PBS)-based blocking buffer containing 10\% goat serum. The samples were then incubated with mouse antiinsulin antibody diluted 1:1,000 in PBS-blocking buffer. Antigen-antibody complexes were visualized with goat anti-rabbit HRP-conjugated streptavidin secondary antibody (Histostain-Plus kit; Zymed Laboratories) diluted 1:1,000 in PBS-blocking buffer. 3,3'-Diaminobenzidine (DAB) substrate (Invitrogen Life Technologies, Carlsbad, CA, USA) and a model GS-690 imaging densitometer (Bio-Rad Laboratories, Hercules, CA, USA) were used to detect insulin proteins.

$R T-P C R$. The frozen fat and liver tissues were chopped with scissors and homogenized in an RNAzol B solution (CS104; Tet-Test, Inc.). The isolated RNA was then determined by UV-spectroscopy. Gene expression was examined by RT-PCR using $5 \mu \mathrm{g}$ of the total RNA from each tissue, and $500 \mathrm{ng}$ of the oligo(dT) primer [(18418-012); Gibco-BRL] was annealed at $70^{\circ} \mathrm{C}$ for $10 \mathrm{~min}$. Complementary DNA, which served as the template for further amplification, was synthesized by adding dATP, dCTP, dGTP and dTTP with 200 units of reverse transcriptase. Ten pmole of the sense and antisense primers were added, and the reaction mixture was then subjected to 25 cycles of amplification. Amplification was carried out in a Perkin-Elmer Thermal Cycler using the following cycles; $30 \mathrm{sec}$ at $94^{\circ} \mathrm{C}, 30 \mathrm{sec}$ at $62^{\circ} \mathrm{C}$ and $45 \mathrm{sec}$ at $72^{\circ} \mathrm{C}$. In each case, the minus-RT controls were included to differentiate the DNA and RNA products. RT-PCR was also performed using the primers specific to $\beta$-actin to ensure the RNA integrity. The primer sequences for VLCAD, MCAD, PPAR- $\gamma, \mathrm{aP} 2$ and leptin were based on a previous study (19). The sequences of the $\beta$-actin sense and antisense primers were, 5'-TGGAA TCCTG TGGCA TCCAT GAAAC-3' and 5'-TAAAA CGCAG CTCAG TAACA GTCCG-3', respectively. The levels of the PCR products were quantified using a Kodak Electrophoresis Documentation and Analysis System 120 on $1 \%$ agarose gels. The experiment was repeated 3 times.

Statistical analysis. The tests for significance between the vehicle- and RLP-treated groups in the OLETF rats were performed using a one-way ANOVA test of variance. In addition, the tests for significance between the LETO and OLETF groups were performed using a post hoc test (all were from SPSS for Windows, Release 10.10, Standard Version; Chicago, IL, USA) of the variance and the significance levels are given in the text. All values are reported as the mean standard deviation (SD). $\mathrm{P}<0.05$ was considered to indicate statistically significant difference.

\section{Results}

Composition of RLP. RLP consisted mainly of carbohydrates (83.22\%) and moisture (8.24\%) and, to a lesser extent, proteins, ash and fat (Fig. 1A). However, following the steaming process, their level was significantly changed into a different concentration. Four components including carbohydrate, protein, ash and fat were slightly increased in RLP, while the moisture was greatly decreased. In particular, the HPLC chromatogram showed a large amount of polyphenolic compounds which have antioxidant activity contained in RLP when compared with the LP (Fig. 1B).

RLP effects on the weight of body and glucose regulation of OLETF rats. The body weights of the LETO and OLETF rats following RLP treatment for 3 weeks were firstly measured to determine if the RLP treatment affects the progress of obesity. The vehicle-treated OLETF rats had a higher body weight than the LETO rats. On the other hand, the body weight was not altered in the RLP-treated group compared to the vehicle-treated group (Fig. 2A). Therefore, the $50 \mathrm{mg} / \mathrm{kg}$ RLP treatment did not work efficiently on the decrease of the body weight to affect the obesity status of OLETF rats.

In addition, the effect of the RLP treatment on the glucose tolerance response was determined by measuring the serum glucose level during the time course of an IPGTT in the 20-week-old OLETF rats using a post-prandial IPGTT. The OLETF rats showed significant impairment in glucose 
$\mathbf{A}$

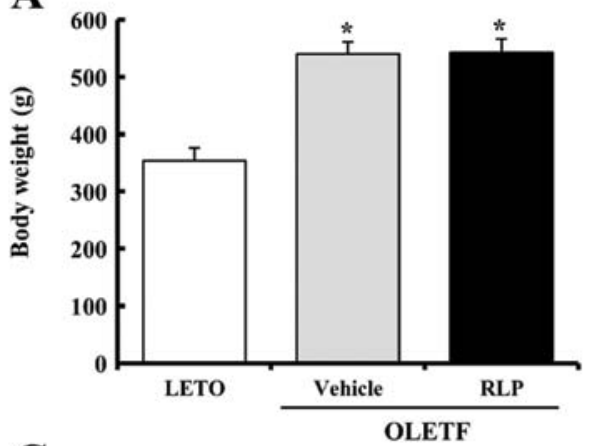

C

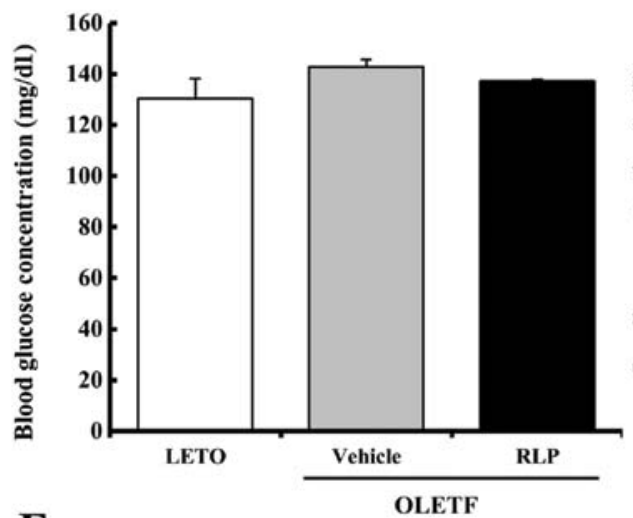

$\mathbf{E}$

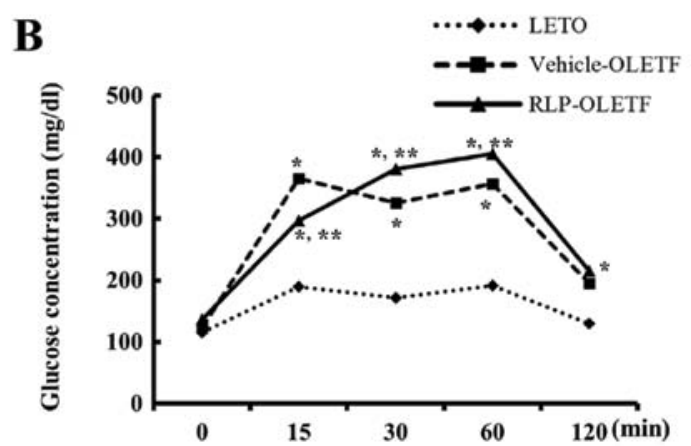

D

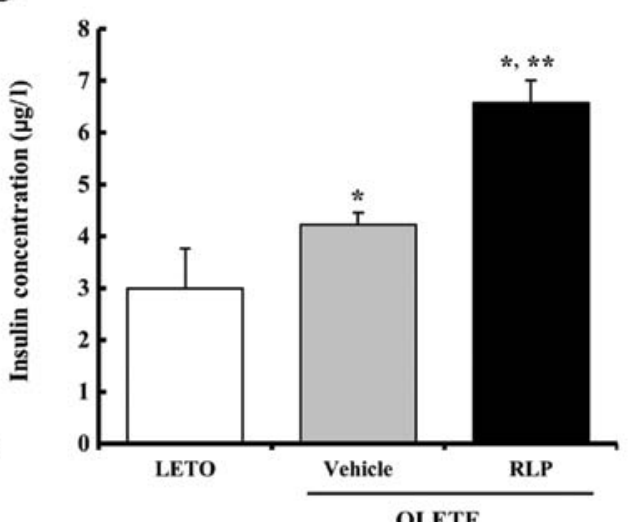

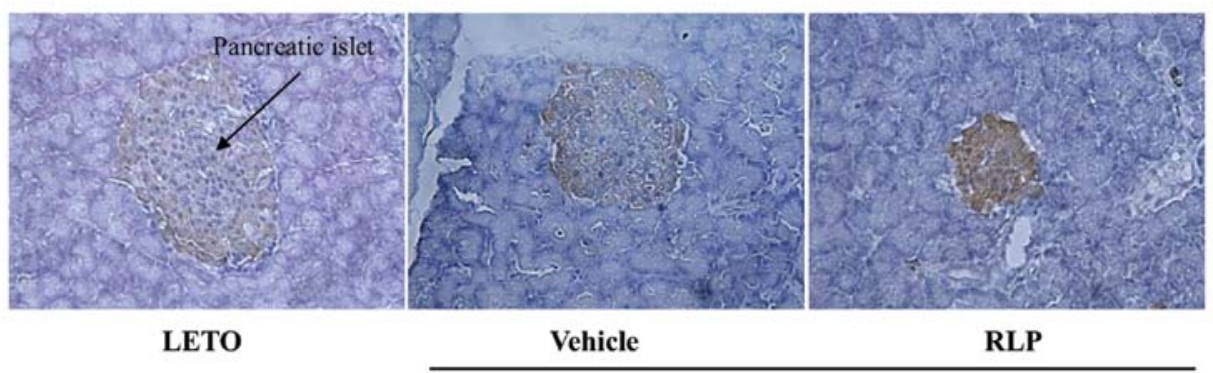

OLETF

Figure 2. Change in body weight, glucose tolerance response, glucose concentration and insulin concentration following RLP treatment. (A) Body weight of the rats in the 3 groups was measured using a chemical balance after the final RLP treatment. (B) The serum glucose level in the IPGTT was monitored over time after an i.p. injection of glucose ( $2 \mathrm{~g} / \mathrm{kg}$ body weight). A difference in the glucose level was observed between the 2 groups 15 min after the injection. Five rats/group were assayed in the IPGTT. (C) The rats were treated orally with RLP (50 mg/kg body weight/day) for 3 weeks. The control group received the vehicle only. On the final day, the glucose concentration was measured in the blood collected from the abdominal vein of rats. In addition, (D) the insulin concentration was detected in the serum using an ELISA kit. This kit has $0.1 \mathrm{ng} / \mathrm{ml}$ of sensitivity and inter-assay coefficient of variation was in the 2.86-5.17 range. (E) Immunostaining analysis for insulin expression. The expression level of insulin was detected in the pancreatic islets of vehicle-treated and AEtLP-treated mice by immunostaining analysis. A high intensity was observed in the pancreatic islets of the RLP-treated rats compared with the vehicle-treated rats at $\times 200$ magnification. Data are reported as the mean $\pm \mathrm{SD}$ from 3 replicates. ${ }^{*} \mathrm{P}<0.05$ is the significance level compared to the LETO rats; ${ }^{* *} \mathrm{P}<0.05$ is the significance level compared to the vehicle-treated OLETF rats.

tolerance compared to the LETO rats during the course of an IPGTT. However, no significant difference was detected between the RLP- and the vehicle-treated group. Only the glucose concentration was lower in the RLP-treated group compared to the vehicle-treated group at the early stage (Fig. 2B). Nevertheless, the blood concentration on the final day of the experiment did not change in the RLP-treated OLETF rats compared to the vehicle-treated rats (Fig. 2C). Regarding insulin, its concentration was significantly higher in the OLETF rats compared to the LETO rats $(\sim 1.43$-fold). In the RLP-treated group, the insulin concentration was increased significantly to $6.4 \mu \mathrm{g} / 1$ (Fig. 2D). Furthermore, a similar pattern was detected in the pancreatic islets using immunostaining analysis (Fig. 2E). These results suggest that RLP treatment can effectively induce an enhancement of insulin secretion ability in the serum of OLETF rats.

RLP effects on the adiponectin and lipid concentration in the serum of OLETF rats. In most animals, adiponectin plays a critical role in controlling the systemic fat catabolism and glucose metabolism (20-22). The adiponectin concentration in the serum of OLETF rats was measured to determine the effect of RLP treatment on the regulation of adiponectin concentration. As shown Fig. 3A, the adiponectin concentra- 
A

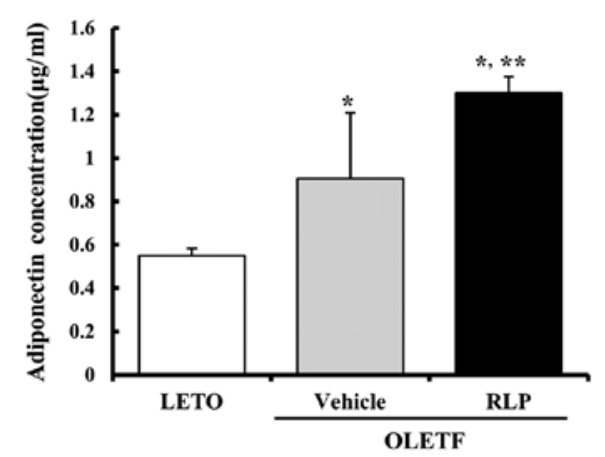

C

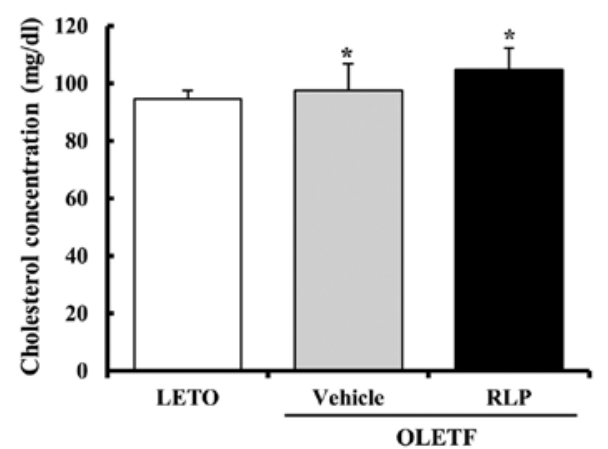

B

D
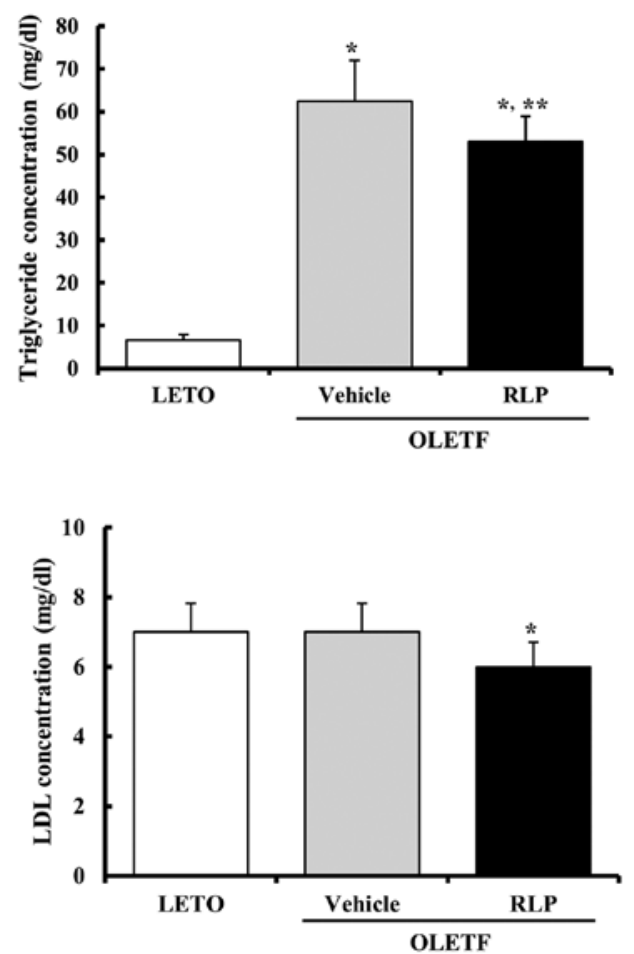

Figure 3. Concentration of (A) adiponectin, (B) triglyceride, (C) cholesterol and (D) LDL cholesterol in the serum. Blood was collected from the abdominal vein of rats and triglyceride, cholesterol and LDL were analyzed in triplicate using a serum biochemical analyzer. Values are reported as the mean \pm SD. ${ }^{*} \mathrm{P}<0.05$ is the significance level compared to the LETO rats; ${ }^{* *} \mathrm{P}<0.05$ is the significance level compared to the vehicle-treated OLETF rats.

tion was increased significantly in the vehicle-treated OLETF rats compared to the LETO rats. In addition, these levels were significantly higher in the RLP-treated rats than in the vehicle-treated OLETF rats. Therefore, these results show that the RLP treatment could induce a strong increase in adiponectin concentration in OLETF rats.

An increase in lipid concentration including triglyceride, cholesterol and LDL is one of the important features observed in obesity (23). The levels of triglyceride, cholesterol and LDL in the serum of OLETF rats were measured to determine if the RLP treatment could induce a change in lipid concentration. Briefly, the triglyceride concentration was higher in the vehicle-treated OLETF rats than in the LETO rats. Following the RLP treatment, the triglyceride concentration was slightly lower in the OLETF rats. However, their level failed to reach that of the LETO rats (Fig. 3B). The LDL concentrations tended to decrease in RLP-treated OLETF rats when compared with the vehicletreated OLETF rats (Fig. 3D), whereas the cholesterol concentration was maintained at a constant level in the RLP-treated group (Fig. 3C). Therefore, RLP treatment may contribute to the reduction of the concentration of some lipids in the sera of OLETF rats.

RLP effects on abdominal fat and adipocyte size of OLETF rats. The fat mass and size of adipocytes were measured under an optical microscope to determine if RLP treatment affects the abdominal fat. Prior to RLP treatment, vehicleOLETF rats showed a higher fat mass than the LETO rats. In particular, the weight of abdominal fat in the vehicle-treated OLETF rats was increased by $675 \%$ compared to the LETO rats. However, there were no significant differences between the vehicle-treated and RLP-treated OLETF rats (Fig. 4A). In addition, the mean size of the adipocytes was $161 \%$ larger in the vehicle-treated OLETF rats compared to the LETO rats. Nevertheless, no changes were detected in the RLP-treated group compared with the vehicle-treated group (Fig. 4A). These results suggest that RLP treatment has little effect on the fat mass and adipocyte size in OLETF rats.

$R L P$ effects on the expression of adipocyte marker genes in abdominal fat. Hypertrophic adipocytes in obese rodents over-express several genes related to the obese metabolism. Therefore, the level of PPAR- $\gamma$, aP2 and leptin expression was measured in the abdominal fat of OLETF rats to determine the effect of the RLP treatment on the expression of adipocyte marker genes. Prior to RLP treatment, the level of PPAR- $\gamma$, aP2 and leptin expression was slightly higher in the OLETF rats than in the LETO rats. On the other hand, in the RLP-treated OLETF rats, the levels of PPAR- $\gamma$ and aP2 expression were decreased by 49 and $39 \%$ compared to the vehicle-treated OLETF rats (Fig. 4B). Leptin expression was even higher in the RLP-treated group. These results suggest that RLP can induce the downregulation of leptin and PPAR- $\gamma$ to regulate the obese metabolism.

$R L P$ effects on the expression of the gene responsible for fatty acid $\beta$-oxidation in abdominal fat. VLCAD and MCAD are 

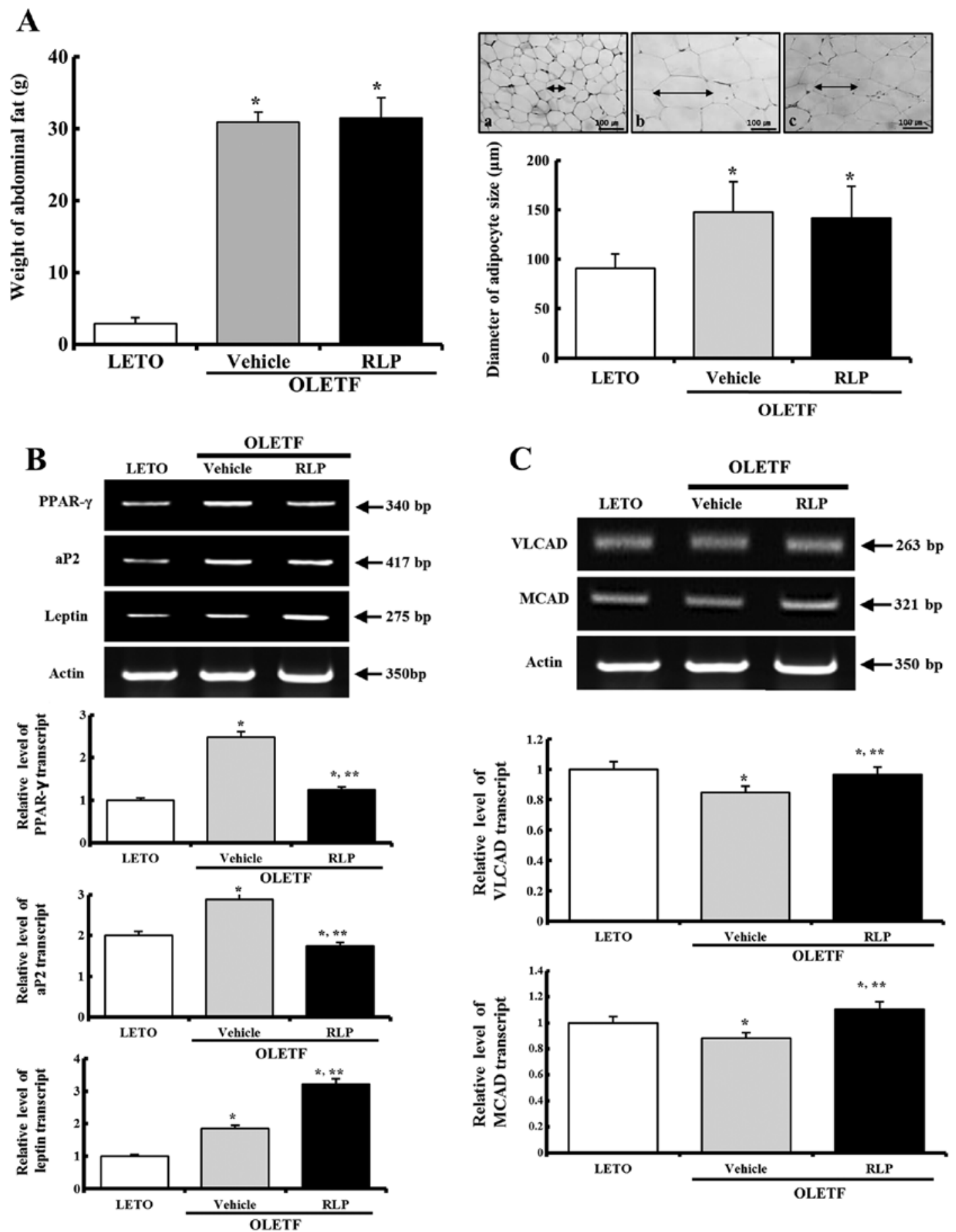

Figure 4. Changes in the fat metabolism in abdominal fat following RLP treatment for 3 weeks. (A) After the final administration, the rats were euthanized in $\mathrm{a} \mathrm{CO}_{2}$ chamber and the abdominal fat was collected from the abdominal cavity. Their weight was measured in triplicate on a chemical balance. The abdominal fat collected from the rats was fixed in $4 \%$ formalin and stained with H\&E solution. The longest diameter of the adipocytes was measured in triplicate using a Leica Application Suite and is regarded as the cell size. (B) Expression of adipocyte marker gene in abdominal fat. The level of PPAR- $\gamma$ (340-bp), aP2 (417-bp) and leptin (275-bp) mRNA was determined by RT-PCR. (C) Expression of the VLCAD and MCAD genes in abdominal fat. The levels of VLCAD (263-bp) and MCAD (321-bp) mRNA were determined by RT-PCR. The $\beta$-actin signal was used as the control and the transcript (350-bp) indicates the RNA loading. The density of transcript was quantified using a Kodak Electrophoresis Documentation and Analysis System 120. Values are reported as the mean \pm SD ${ }^{*} \mathrm{P}<0.05$ is the significance level compared to the LETO rats; ${ }^{* *} \mathrm{P}<0.05$ is the significance level compared to the vehicle-treated OLETF rats.

important enzymes for fatty acid oxidation (24). The levels of these enzymes were detected in the abdominal fat by RT-PCR to determine the effect of RLP treatment on the expression of VLCAD and MCAD. In the obese condition, the expression of these enzymes was slightly lower compared to the normal condition. On the other hand, the RLP-treated OLETF rats showed an increase in VLCAD and MCAD expression compared to the vehicle-treated OLETF rats (Fig. 4C). Therefore, these results show that the expression of VLCAD and MCAD was significantly increased by the RLP treatment for 3 weeks.

RLP effects on the fatty liver formation and expression of the gene responsible for fatty acid $\beta$-oxidation in the 

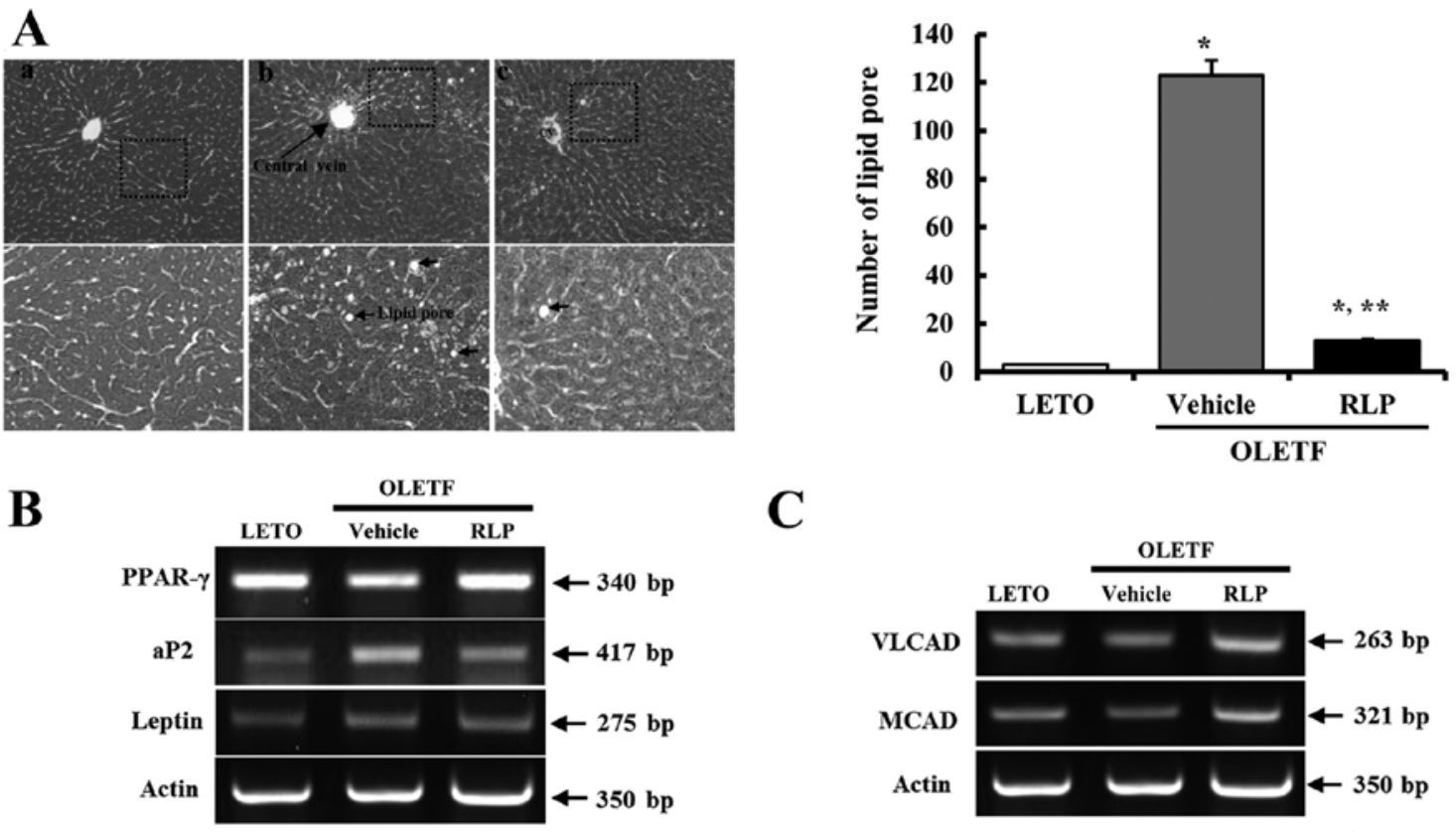

C
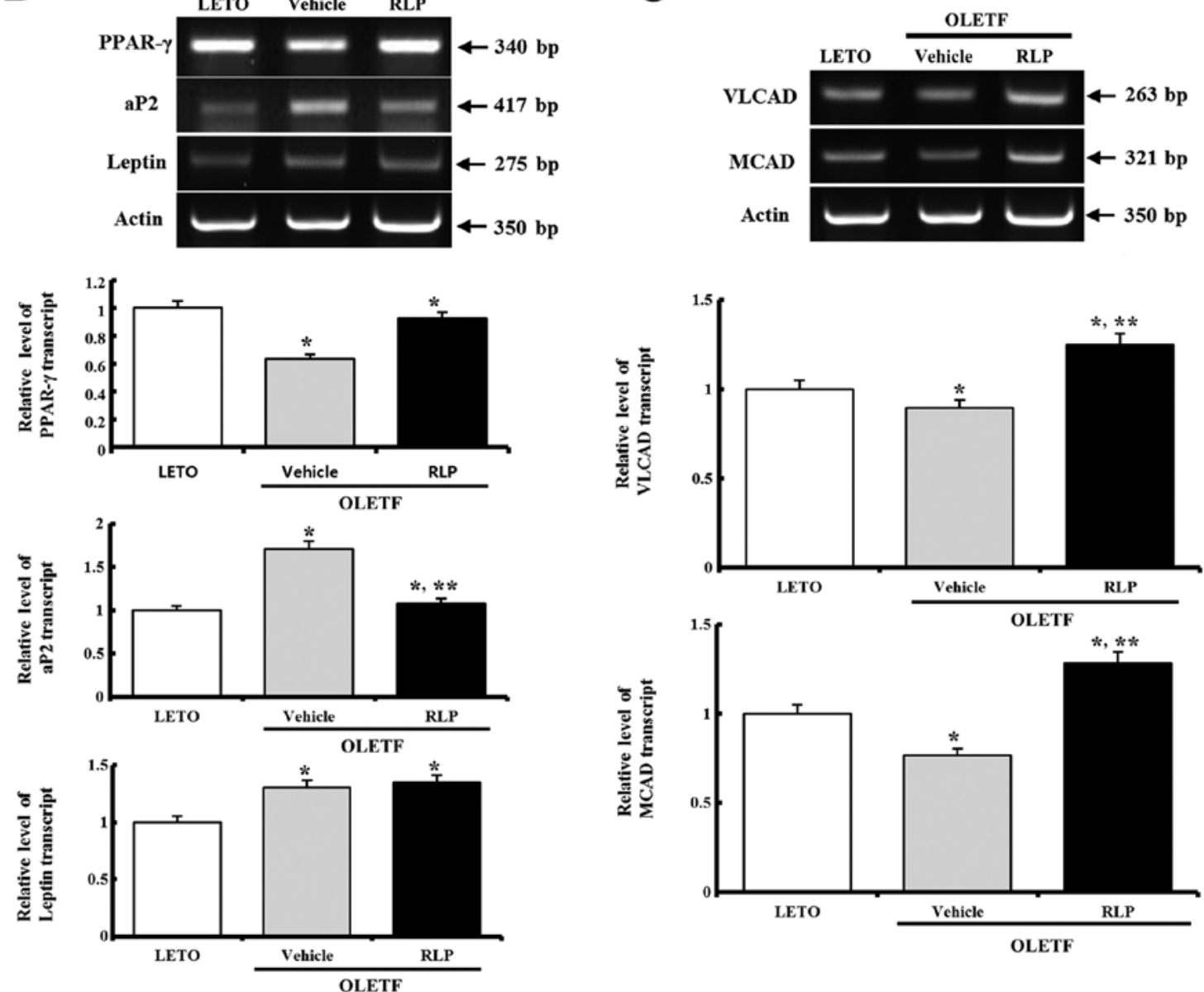

Figure 5. Changes in the fat metabolism in the liver following RLP treatment for 3 weeks. (A) After the final administration, the rats are euthanized in a $\mathrm{CO}_{2}$ chamber and the liver was collected from the abdominal cavity. The liver collected from the rats was fixed in $4 \%$ formalin and stained with $\mathrm{H} \& \mathrm{E}$ solution. The number of lipid pore was counted in triplicate using a Leica Application Suite. (B) Expression of the adipocyte marker gene in liver tissue. The level of PPAR- $\gamma$ (340-bp), aP2 (417-bp) and leptin (275-bp) mRNA was measured by RT-PCR. (C) Expression of the VLCAD and MCAD genes in liver tissue. The levels of VLCAD (263-bp) and MCAD (321-bp) mRNA were detected by RT-PCR. The $\beta$-actin signal was used as the control and the transcript (350-bp) indicates the RNA loading. The density of the transcript was quantified using a Kodak Electrophoresis Documentation and Analysis System 120 . Values are reported as the mean $\pm \mathrm{SD}$. " $\mathrm{P}<0.05$ is the significance level compared to the LETO rats; " $\mathrm{P}<0.05$ is the significance level compared to the vehicle-treated OLETF rats.

liver. To determine if the RLP treatment affects the lipid metabolism of the liver, the alteration of fat accumulation and gene expression for fatty acid $\beta$-oxidation was observed in the liver tissue. Fatty liver formation was higher in the OLETF rats compared to the LETO rats. However, following RLP treatment, these levels in the OLETF rats decreased significantly to near the level of the LETO rats (Fig. 5A). In addition, of the 3 adipocyte marker genes, aP2 and leptin expression was increased significantly in the liver of the OLETF rats, even though PPAR- $\gamma$ was decreased in the OLETF rats. However, these alteration patterns were transformed into different ones. The expression levels of the PPAR- $\gamma$ and aP2 genes in RLP-treated OLETF rats were recovered to those measured in the LETO rats, but leptin expression was increased to more than that observed in the LETO rats (Fig. 5B). Therefore, RLP can induce the regulation of PPAR- $\gamma, \mathrm{aP} 2$ and leptin expressions to control the obese metabolism. 
In the case of gene expression analysis for fatty acid $\beta$-oxidation, the expression level of the VLCAD and MCAD genes were significantly lower in the OLETF rats than in the LETO rats. However, these levels were higher in the RLP-treated OLETF rats than in the vehicle-treated OLETF rats (Fig. 5C). Therefore, RLP treatment can induce gene expression for fatty acid $\beta$-oxidation in the liver of OLETF rats.

\section{Discussion}

LP and RLP have attracted considerable attention as novel therapeutic drugs $(3,8,16)$. As part of the research into the development of drugs for obesity and diabetes, this study examined the effects of RLP on the glucose and fat metabolism using a type II diabetes model with obesity. The results suggest that RLP treatment can induce a decrease in the symptoms developed in diabetes and obesity.

The OLETF rats used in this study were first developed by the selective breeding of rats showing spontaneous obesity in an outbred colony of Long-Evans rats at Otsuka Pharmaceuticals (25). The OLETF rats consumed 30\% more food than the LETO rats due to the fact that these rats have a food intake disorder induced by a cholecystokinin (CCK) 1 receptor defect. Therefore, they become 35\% more obese than LETO rats (26). On the other hand, the OLETF rats showed different properties to the other obesity rat model on fat deposition. Most of the fat in the OLETF rats was deposited in the intra-abdominal or visceral areas, whereas the fat in Zucker rats was deposited in subcutaneous areas (27). In this study, the body weight of the OLETF rats was 25\% higher than in the LETO rats. In addition, the abdominal fat was increased significantly in the OLETF rats. Therefore, the OLETF rats used in this experiment showed spontaneous obesity.

There are few reports on the relationship between LP and abdominal fat. In particular, Gyeongshingangjeehwan (GGEx), which is comprised of Liriope platyphylla F.T. Wang and T. Tang (Liliaceae), Platycodongrandiflorum A. DC. (Campanulaceae), Schisandrachinensis K. Koch (Magnoliaceae) and Ephedra sinica Stapf (Ephedraceae), exhibited anti-obesity abilities. Compared to the obese OLETF control rats, administration of GGEx for 8 weeks significantly decreased the food intake and plasma leptin levels as well as the body weight gain and abdominal fat in OLETF rats (6). In the present study, a significant change in the body weight and abdominal fat was not detected in the vehicle- and RLP-treated rats for 3 weeks (Figs. 2 and 4). The main reasons for these differences were the administration period and the composition of the drug; however, further research is required.

Insulin produced by pancreatic $\beta$-cells plays a key role in regulating the carbohydrate and fat metabolism in an animal body. Insulin induces glucose uptake from the blood to the cells in the liver, muscle and fat tissue, and stores it as glycogen in the liver and muscle $(28,29)$. In vitro analysis showed a dramatic increase in insulin secretion in RLP-treated INS cells compared to the vehicle-treated group (16). Therefore, this study aimed to confirm these results in an animal model for type II diabetes with obesity. As shown in Fig. 2D, the insulin concentration was significantly higher in the RLP-treated OLETF rats than in the vehicle-treated OLETF rats. Therefore, these results provide strong evidence that RLP treatment can induce an increase in insulin concentration in OLETF rats.

Adiponectin is considered an important hormone that is involved in the process of regulating the fatty acid catabolism and glucose regulation (22). This hormone also contributed to suppress the metabolic disorders including diabetes, obesity, atherosclerosis and non-alcoholic fatty liver disease $(22,30)$. Adiponectin is maintained at approximately $5-10 \mu \mathrm{g} / \mathrm{ml}$ in the bloodstream of humans and the level is lower in diabetics than non-diabetics. In healthy human adults, the concentration of this hormone was inversely related to the body fat mass. The decrease in body weight induced a significant increase in adiponectin concentration (31). In the present study, the concentration of adiponectin was significantly higher in the OLETF rats. This concentration was also increased following RLP treatment. This differs from previous findings in that the adiponectin level was inversely related to the body fat mass in healthy humans. This difference may be caused by a metabolic disorder including a CCK1 receptor defect in OLETF rats.

Under diabetic conditions induced by the administration of streptozotocin, the total cholesterol, HDL, triglycerides, creatinine and urea concentrations were significantly higher than under non-diabetic conditions (23). Of these lipids, the circulating triglycerides were decreased by GGEx treatment for 8 weeks in the OLETF rats (6). Our findings from RLP treatment for 3 weeks are in agreement with previous studies, even though the rate of decrease was different. These results suggest that LP can effectively induce a decrease of triglycerides in serum, regardless of the administration period and concentration of LP used.

Acyl-CoA dehydrogenase is a catalytic enzyme that participates in the initial step in each cycle of fatty acid oxidation in the mitochondria of cells. This enzyme can be classified into 3 groups based on their specificity for long-, medium- and short-chain fatty acid substrates (24). Of the 3 enzymes, the levels of VLCAD and MCAD were significantly lower in the visceral white adipose tissue of the OLETF rats compared to the LETO rats and some polyherbal drugs and GGEx could recover the levels (19). VLCAD and MCAD were also expressed in the liver of rats and play a key role in fatty acid oxidation. Furthermore, their expression was regulated by several diet types. Following a high fat diet, the mRNA level of the VLCAD gene was increased considerably in the liver of rats compared to that in the liver of rats fed low fat chow, whereas there was no change in the mRNA level of the VLCAD gene in the muscle of the same rats (32). In addition, $10 \% \mathrm{TG}+4 \% \alpha$-linolenic acid-rich diacylglycerol diets for a period of 1 month induced an upregulation of $\beta$-oxidation activity, as well as the acyl-CoA oxidase (ACO) and MCAD mRNA levels (33). In this study, the levels of VLCAD and MCAD expression in the abdominal fat and liver of OLETF rats were also increased by the RLP treatment for 3 weeks, even though the increase rate was different in both. This study suggests that RLP treatment can induce VLCAD and MCAD expression in the abdominal fat and liver tissue of OLETF rats. 
Our results suggest that RLP induces an increase in the insulin and adiponectin concentration, as well as a significant change in the lipid concentration and expression of adipocyte marker genes in the diabetic and obese OLETF rats. Therefore, RLP may be considered a new potential treatment for obesity through its ability to reduce the lipid metabolism.

\section{Acknowledgements}

The authors thank Jin Hyang Hwang, the animal technicians, for directing the Animal Facility and Care at the Laboratory Animal Resources Center. This study was supported by grants to Dr Dae Youn Hwang from the Korea Institute of Planning Evaluation for Technology of Food, Agriculture, Forestry and Fisheries (110119-3).

\section{References}

1. Huh MK, Huh HW, Choi JS and Lee BK: Genetic diversity and population structure of Liriope platyphylla (Liliaceae) in Korea. J Life Sci 17: 328-333, 2007.

2. Lee YC, Lee JC, Seo YB and Kook YB: Liriopis tuber inhibits OVA-induced airway inflammation and bronchial hyperresponsiveness in murine model of asthma. J Ethnopharmacol 101: 144-152, 2005.

3. Choi SB, Wha JD and Park S: The insulin sensitizing effect of homoisoflavone-enriched fraction in Liriope platyphylla Wang et Tang via PI3-kinase pathway. Life Sci 75: 2653-2664, 2004.

4. Kim SW, Chang IM and Oh KB: Inhibition of the bacterial surface protein anchoring transpeptidase sortase by medicinal plants. Biosci Biotechnol Biochem 66: 2751-2754, 2002.

5. Hur J, Lee P, Kim J, Kim AJ, Kim H and Kim SY: Induction of nerve growth factor by a butanol fraction of Liriope platyphylla in C6 and primary astrocyte cells. Biol Pharm Bull 27: 1257-1260, 2004.

6. Jeong S, Chae K, Jung YS, Rho YH, Lee J, Ha J, Yoon KH, Kim GC, Oh KS, Shin SS and Yoon M: The Korean traditional medicine Gyeongshingangjeehwan inhibits obesity through the regulation of leptin and PPARalpha action in OLETF rats J Ethnopharmacol 119: 245-251, 2008.

7. Hur J, Lee P, Moon E, Kang I, Kim SH, Oh MS and Kim SY: Neurite outgrowth induced by spicatoside A, a steroidal saponin, via the tyrosine kinase A receptor pathway. Eur J Pharmacol 620: 9-15, 2009

8. Lee YK, Kim JE, Nam SH, Goo JS, Choi SI, Choi YH, Bae CJ, Woo JM, Cho JS and Hwang DY: Differential regulation of the biosynthesis of glucose transporters by the PI3-K and MAPK pathways of insulin signaling by treatment with novel compounds from Liriope platyphylla. Int J Mol Med 27: 319-327, 2011.

9. Kim JE, Nam SH, Choi SI, Hwang IS, Lee HR, Jang MJ, Lee CY, Soon HJ, Lee HS, Kim HS, et al: Aqueous extracts of Liriope platyphylla are tightly-regulated by insulin secretion from pancreatic islets and by increased glucose uptake through glucose transporters expressed in liver hepatocytes. Biomol Ther 19: 348-356, 2011.

10. Kim K and Kim HY: Korean red ginseng stimulates insulin release from isolated rat pancreatic islets. J Ethnopharmacol 120: 190-195, 2008

11. Lu JM, Yao Q and Chen C: Ginseng compounds: an update on their molecular mechanisms and medical applications. Curr Vasc Pharmacol 7: 293-302, 2009.

12. Ng TB: Pharmacological activity of sanchi ginseng (Panax notoginseng). J Pharm Pharmacol 58: 1007-1019, 2006.

13. Kiefer D and Pantuso T: Panax ginseng. Am Fam Physician 68: 1539-1542, 2003.

14. Baek NI, Kim DS, Lee YH, Park JD, Lee CB and Kim SI: Ginsenoside Rh4, a genuine dammarane glycoside from Korean red ginseng. Planta Med 62: 86-87, 1996.
15. Yun TK, Lee YS, Kwon KH and Choi KJ: Saponin contents and anticarcinogenic effects of ginseng depending on types and ages in mice. Acta Pharmacol Sin 17: 293-298, 1996.

16. Choi SI, Lee HR, Goo JS, Kim JE, Nam SH, Hwang IS, Lee YJ, Prak SH, Lee HS, Lee JS, et al: Effects of steaming time and frequency for manufactured red Liriope platyphylla on the insulin secretion ability and insulin receptor signaling pathway. Lab Anim Res 27: 117-126, 2011.

17. Kim SD, Ku YS, Lee IZ, Kim ID and Youn KS: Gerneral components and sensory evaluation of hot water extract from Liriopis Tuber. J Korean Soc Food Sci Nutr 30: 20-24, 2001.

18. Kim JE, Lee YK, Nam SH, Choi SI, Goo JS, Jang MJ, Lee HS Son HJ, Lee CY and Hwang DY: The symptoms of atopic dermatitis in $\mathrm{NC} / \mathrm{Nga}$ mice were significantly relieved by the water extract of Liriope platyphylla. Lab Anim Res 26: 377-384, 2010.

19. Shin SS, Jung YS, Yoon KH, Choi S, Hong Y, Park D, Lee H, Seo BI, Lee HY and Yoon M: The Korean traditional medicine gyeongshingangjeehwan inhibits adipocyte hypertrophy and visceral adipose tissue accumulation by activating PPARalpha actions in rat white adipose tissues. J Ethnopharmacol 127: 47-54, 2010.

20. Arita Y, Kihara S, Ouchi N, Takahashi M, Maeda K, Miyagawa J, Hotta K, Shimomura I, Nakamura T, Miyaoka K, et al: Paradoxical decrease of an adipose-specific protein, adiponectin, in obesity. Biochem Biophys Res Commun 257: 79-83, 1999.

21. Yang WS, Lee WJ, Funahashi T, Tanaka S, Matsuzawa Y, Chao CL, Chen CL, Tai TY and Chuang LM: Weight reduction increases plasma levels of an adipose-derived anti-inflammatory protein, adiponectin. J Clin Endocrinol Metab 86: 3815-3819, 2001.

22. Diez JJ and Iglesias P: The role of the novel adipocyte-derived hormone adiponectin in human disease. Eur J Endocrinol 148: 293-300, 2003.

23. Roy S, Dontamalla SK, Mondru AK, Sannigrahi S and Veerareddy PR: Downregulation of apoptosis and modulation of TGF- $\beta 1$ by sodium selenate prevents streptozotocin-induced diabetic rat renal impairment. Biol Trace Elem Res 139: 55-71, 2011.

24. Kim JJ, Wang M and Paschke R: Crystal structures of mediumchain acyl-CoA dehydrogenase from pig liver mitochondria with and without substrate. Proc Natl Acad Sci USA 90: 7523-7527, 1993.

25. Kawano K, Hirashima T, Mori S and Natori T: OLETF (Otsuka Long-Evans Tokushima Fatty) rat: a new NIDDM rat strain. Diabetes Res Clin Pract 24: S317-S320, 1994.

26. Moran TH: Unraveling the obesity of OLETF rats. Physiol Behav 94: 71-78, 2008.

27. Mori Y and Ikeda Y: Similarity and dissimilarity between the OLETF rats and obese subjects with NIDDM. In: Obesity and NIDMM. Lessons from the OLETF Rat. Shima K (ed). Elsevier Science BV, Amsterdam, pp237-244, 1999.

28. Le Roith D and Zick Y: Recent advances in our understanding of insulin action and insulin resistance. Diabetes Care 24: 588-597, 2001.

29. Taha C and Klip A: The insulin signaling pathway. J Membr Biol 169: 1-12, 1999.

30. Ukkola O and Santaniemi M: Adiponectin: a link between excess adiposity and associated comorbidities? J Mol Med 80: 696-702, 2002.

31. Coppola A, Marfella R, Coppola L, Tagliamonte E, Fontana D, Liguori E, Cirillo T, Cafiero M, Natale S and Astarita C: Effect of weight loss on coronary circulation and adiponectin levels in obese women. Int J Cardiol 134: 414-416, 2009.

32. Ji H and Friedman MI: Reduced capacity for fatty acid oxidation in rats with inherited susceptibility to diet-induced obesity. Metabolism 56: 1124-1130, 2007.

33. Murase T, Aoki M and Tokimitsu I: Supplementation with alpha-linolenic acid-rich diacylglycerol suppresses fatty liver formation accompanied by an up-regulation of beta-oxidation in Zucker fatty rats. Biochim Biophys Acta 1733: 224-231, 2005. 